\title{
Numerical Investigations of Two-phase Flows through Enhanced Microchannels
}

\author{
A. K. Chandra, ${ }^{a}$ K. Kishor, ${ }^{a}$ P. K. Mishra, ${ }^{b}$ and M. S. Alam ${ }^{a, *}$ \\ aDepartment of Chemical Engineering \\ ${ }^{b}$ Department of Mechanical Engineering \\ Motilal Nehru National Institute of Technology, Allahabad, India
}

doi: 10.15255/CABEQ.2015.2289

Original scientific paper

Received: August 15, 2015

Accepted: June 1, 2016

Microfluidic devices are quite important for process industries, as these devices can intensify heat and mass transfer in two-phase reaction systems. Two-phase reaction systems, such as gas-liquid and liquid-liquid reactions with certain limitations have already been carried out in microfluidic systems by a few authors. However, these concepts are still under development and a detailed understanding of the hydrodynamics involve is required. Hydrodynamics studies are inherently crucial to provide precise reaction conditions and identify asymptotic performance limits. In the present work, Computational Fluid Dynamics (CFD) simulation was carried out to investigate the hydrodynamics involved in the T-junction enhanced microchannel. The slug formation, slug size, slug shape, and pressure drop in the enhanced microchannel were predicted using the volume of fluid (VOF) for water-cyclohexane system. The effects of obstruction spacing on pressure drop, slug lengths, and mixing within the slug were also examined. This study revealed that mixing enhances tremendously within the slug and at the interface in the enhanced microchannel, but with slightly greater pressure drop. However, an increase in obstruction spacing affects the slug formation, unit slug length, and pressure drop.

Key words:

microchannel, liquid-liquid flows, pressure drop, slug flow, enhanced microchannel

\section{Introduction}

Microfluidic systems that involve gas-liquid or liquid-liquid two-phase operations have been studied intensively as the heat and mass transfer in these devices are notably higher compared to conventional devices. In fact, this enhancement is possible in the microfluidic system due to well-defined flow patterns and very high interfacial area. Further, high surface to volume ratio and small volume of working fluid in microfluidic systems speed up the chemical reaction and improve the product yield. In addition, the shearing motion within fluid segments produces vortex flow patterns in the channel, which enhances mixing within segments and improves mass transfer across the interface, providing uniform mass and temperature distributions in the channel.

In literature, several experimental and numerical works pertaining to the hydrodynamics of gas-liquid flow in microchannels are available; however, there is scarcity of such work on immiscible liquid-liquid two-phase systems in microfluidic devices. Liquid-liquid two-phase systems behave quite differently from gas-liquid systems. There-

"Corresponding author (email: alamsiraj@gmail.com) fore, results for gas-liquid systems cannot be generalized for liquid-liquid systems. Due to these facts, recently, attention has been given to immiscible liquid-liquid microfluidic systems with a focus on intensification of mass transfer and reaction by dispersing one phase into the other as droplets. Numerous studies concerning the flow patterns of liquid-liquid systems in microchannels have indicated that the flow pattern can be controlled by the channel geometry, interfacial properties of fluids and channel surface, as well as the flow rate of two immiscible liquids ${ }^{11-13}$

The flow pattern and droplets formation mechanism of dispersed phase have been experimentally studied by several authors, who have discussed the flow regimes in microchannels viz. mono-dispersed drop flow, slug flow, parallel flow based on the Weber numbers of fluid phases ${ }^{14-17}$. The free surface simulation has been carried out by Kashid et al. ${ }^{18}$ to understand the mechanism of slug flow generation. In an another experimental study, the droplets formation of the oil-water two-phase system has been investigated in a microchannel ${ }^{19}$. Thorsen et al..$^{20}$ investigated the influence of the velocity ratio of the two phases on the droplet size in a T-shaped microchannel. Similar studies have also been carried out for oil-water systems in a microfluidic T-junction 
channel by Garstecki et al..$^{21}$ and a correlation (scaling law) was proposed to predict the slug length as a function of the ratio of dispersed to continuous phase flow as per scaling law, which is given below:

$$
\frac{L_{d}}{W_{\text {oil }}}=1+\alpha \frac{Q_{\text {water }}}{Q_{\text {oil }}}
$$

The accuracy and range of applicability of the scaling law was verified experimentally for flow rate range: $10^{-2}$ to $1 \mu \mathrm{L} \mathrm{s}^{-1}$, ratio $Q_{\text {water }} / Q_{\text {oil }}(0.1-$ $10)$, $\mu$ oil $\left(0.01-0.1 \mathrm{~kg} \mathrm{~m}^{-1} \mathrm{~s}^{-1}\right)$, and surface tension in a microchannel $100 \mu \mathrm{m}$. The effect of flow velocity on the slug size has also been experimentally studied and verified by Tice et al. ${ }^{22}$

Further, the effects of various operating conditions on the flow regimes, slug size, interfacial area, and pressure drop in Y-junction capillaries were experimentally and numerically studied for liquid-liquid two-phase flow ${ }^{23}$. Their numerical study concluded that the surface tension and wall adhesion plays an important role in slug generation. In an another study, the effect of mixing element ( $\mathrm{T}$ and $\mathrm{Y})$ on the two-phase flow patterns was discussed and reported ${ }^{5}$. The authors reported that the pressure drop across the mixing element contributed significantly in the overall pressure drop, and it was also reported that the pressure drop in the Y-junction was lower compared to T-junction mixing elements due to lower frictional losses. Kashid et al. ${ }^{24}$ developed a VOF-based CFD methodology to investigate the slug flow generation in a T-type mixing element. In their work, a stable liquid wall film was considered. These results were validated with the experimental results of Tice et al..$^{22}$

Yong et al. ${ }^{25}$ numerically studied the immiscible kerosene-water two-phase flows in a T-junction microchannel using a Lattice Boltzmann (LB) method. This result quantitatively and qualitatively verified the experimental results of Zhao et al. ${ }^{14-16}$ and justified the effectiveness of the numerical method. Further, the effect of interfacial tension and contact angle on the flow patterns and droplet shapes were investigated, and highlighted the numerical results based on the improved two-phase LB model.

In this investigation, a VOF-based CFD methodology was developed to investigate the slug flow generation and pressure drop in liquid-liquid twophase flow in a T-junction square enhanced microchannel. The computational methodology along with the underlying physics is discussed to understand the slug flow generation mechanism in order to develop an understanding of the fluid dynamics in the enhanced channel. In addition, the effect of channel geometry (obstruction spacing and height) on slug shapes, length, and pressure drop was stud- ied, and the predictions verified using the measurements of Kashid M. N. ${ }^{26}$ The effect of dispersed phase flow rate was also investigated.

\section{Model development}

In the present work, a 3D T-junction square enhanced microchannel was chosen to study the hydrodynamics under slug flow conditions. The schematic diagram of the microchannel geometry with boundary conditions is shown in Fig. 1. The width and height of the main channels and the side entry were the same and each was $1 \mathrm{~mm}$ in size. The lengths of the side entry and the main channel were $2 \mathrm{~mm}$ and $50 \mathrm{~mm}$, respectively. The T-junction enhanced microchannels consisted of triangular shaped obstruction elements of heights $(\varepsilon)$ 0.05, 0.1, 0.15 and $0.2 \mathrm{~mm}$, and were placed on the inner side of the channel walls, which were perpendicular to the flow direction, as shown in Fig. 1. These obstructions were placed in a staggered manner, and the distance between the obstructions varied from 0.5 to $5 \mathrm{~mm}$. Water and cyclohexane were used as working fluids, and the physical properties of these fluids are given in Table 1. The streams of water and cyclohexane were fed separately through two inlet points into the channel. The superficial velocity of water as well as superficial velocity of cyclohexane varied from 0.005 to $0.07 \mathrm{~m} \mathrm{~s}^{-1}$.

\section{Hydrodynamic model}

In the present work, unsteady state flow behaviors were considered in the channel with the following assumptions:

- Fluids in both the phases are Newtonian, viscous, and incompressible.

- Physical properties of the fluids are constant and unaffected by the channel geometry.

- Laminar flow for both the phases.

- Gravity effects are negligible.

- The mean velocity of both the phases within the slug unit is the same. This assumption may fail for very low capillary number in the range of $10^{-3}<$ $<\mathrm{C}_{\mathrm{a}}<1$.

The isothermal two-phase flow of Newtonian fluids of constant density, which are immiscible on the molecular scale, was described using the one-fluid approach. The volume fraction $\phi$ allows tracking the phase: it equals zero in the continuous phase, and one in the dispersed phase. The mass, momentum, and volume fraction conservation equation for the two-phase flow through the domain are represented as: 


$$
\nabla \bullet u=0
$$

$$
\frac{\partial u}{\partial t}+\nabla \bullet(u u)=-(\nabla p)+\nabla \bullet\left[\mu\left(\nabla \vec{u}+\nabla \vec{u}^{T}\right)\right]-\sigma \kappa_{n}\left[\frac{\varphi_{1} \rho_{1}+\varphi_{2} \rho_{2}}{\left(\rho_{1}+\rho_{2}\right) / 2}\right]
$$

where, $\sigma$ is the surface tension and $\kappa_{n}$ is the surface curvature at the fluid interface computed from the divergence of the unit surface normal $\hat{n}$ as

$$
\kappa_{n}=\nabla \cdot \hat{n} \frac{1}{|n|}\left[\left(\frac{n}{|n|} \cdot \nabla\right)|n|-(\nabla \cdot n)\right]
$$

and

$$
\widehat{n}=\frac{n}{|n|}, n=\nabla \varphi
$$

The interface between the two phases was traced by solving the continuity equation for the volume fraction function:

$$
\frac{\partial \varphi}{\partial t}+u \bullet \nabla \varphi=0
$$

The momentum equation, Eq. (3) is dependent on the volume fractions of both phases through the properties density $(\rho)$ and viscosity $(\mu)$. For a twophase system, if the phases are represented by the subscripts 1 and 2 and the volume fraction of the phase 2 is known, the density and viscosity in each cell are given by Eqs. (7) and (8):

$$
\begin{aligned}
& \rho=\varphi_{2} \rho_{2}+\left(1-\varphi_{1}\right) \rho_{1} \\
& \mu=\varphi_{2} \mu_{2}+\left(1-\varphi_{1}\right) \mu_{1}
\end{aligned}
$$

\section{Numerical simulation}

Various methods are available to model immiscible liquid-liquid two-phase flows in a microchannel, such as volume of fluid (VOF), marker particle, lattice Boltzmann, level set and front tracking. VOF and level set method are the most common implicit free surface reconstruction methods, wherein VOF methodology is relatively simple and extensively used for many applications. The VOF methodology relies on the fact that two or more phases are not interpenetrating, and for each additional phase, the volume fraction of the phase is added in the computational cell. The sum of volume fraction of all the phases is unity in each control volume. Further, VOF methodology has several advantages such as

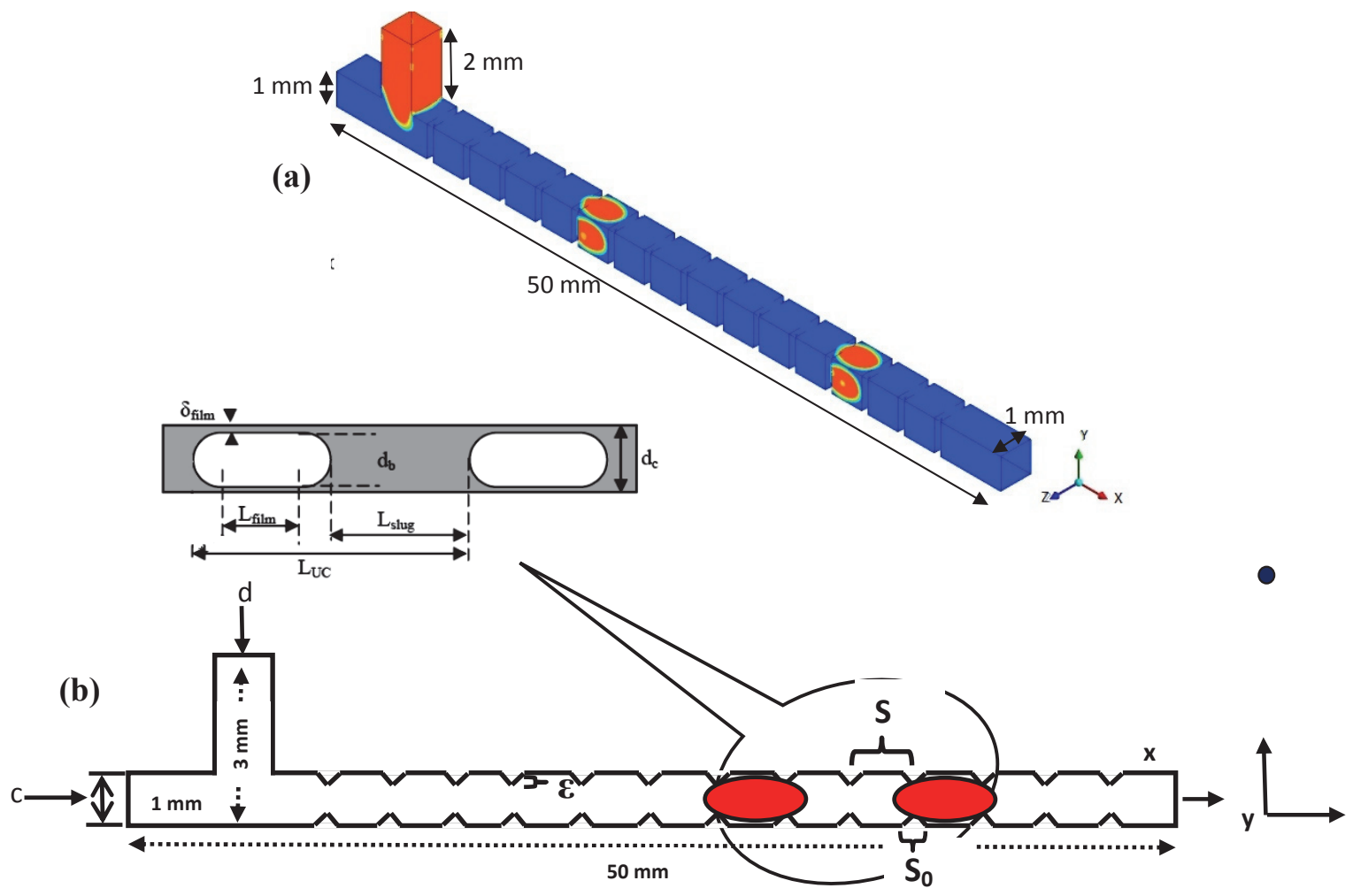

Fig. 1 - Schematic diagram of enhanced microchannel (a) isometric view (b) side view 
reasonable accuracy, relatively simple application, and it can be used to solve highly complex free surface flows. In addition, it can also be accurately applied to boundary fitted grids, and it accommodates breaking and forming of interfaces.

A 3D T-junction enhanced microchannel geometry was built and meshed with the pre-processor Design Modular (ANSYS Inc.), then imported into FLUENT for slug flow calculation. A structured mesh using triangular elements was used to generate mesh, and refined near the wall and in the mixing zone to capture the fluid flow accurately. The grid independency test was performed for the enhanced microchannel to use an appropriate grid for slug flow development. The grid size was reduced until the difference in the key parameters, $u$ and $p$ was less than $0.5 \%$ for two consecutive grid sizes. Computational cells containing $2.85 \times 10^{5}, 4.91 \times 10^{5}$,

Table 1 - Physical properties of fluids used, and simulation parameters

\begin{tabular}{lll}
\hline \multicolumn{1}{c}{ Property/parameter } & \multicolumn{1}{c}{ Fluid } & \multicolumn{1}{c}{ Value } \\
\hline Density $(\rho)\left(\mathrm{kg} \mathrm{m}^{-3}\right)$ & $\begin{array}{l}\text { Cyclohexane } \\
\text { Water }\end{array}$ & 780 \\
Dynamic viscosity $(\mu)\left(\mathrm{kg} \mathrm{m}^{-1} \mathrm{~s}^{-1}\right)$ & $\begin{array}{l}\text { Cyclohexane } \\
\text { Water }\end{array}$ & 998.2 \\
Interfacial tension $(\sigma)\left(\mathrm{N} \mathrm{m}^{-1}\right)$ & Cyclohexane/water & $9.82 \cdot 10^{-4}$ \\
Dispersed phase fraction $(\phi)$ & & $1.003 \cdot 10^{-3}$ \\
Dimension of microchannel & & 0.05 \\
Length of microchannel $(\mathrm{mm})$ & 0.5 \\
Obstruction spacing $(\mathrm{mm})$ & Width $=1 \mathrm{~mm}$, Height $=1 \mathrm{~mm}$ \\
Obstruction height $\varepsilon(\mathrm{mm})$ & 50 \\
Obstruction width $s_{0}(\mathrm{~mm})$ & $0.5,0.75,1,1.25,2.5$ and 5 \\
Minimum time step $(\mathrm{s})$ & $0.05,0.1,0.15,0.2$ \\
Maximum time step $(\mathrm{s})$ & 0.2 \\
Mesh size $(\mu \mathrm{m})$ & $1 \cdot 10^{-8}$ \\
\hline
\end{tabular}

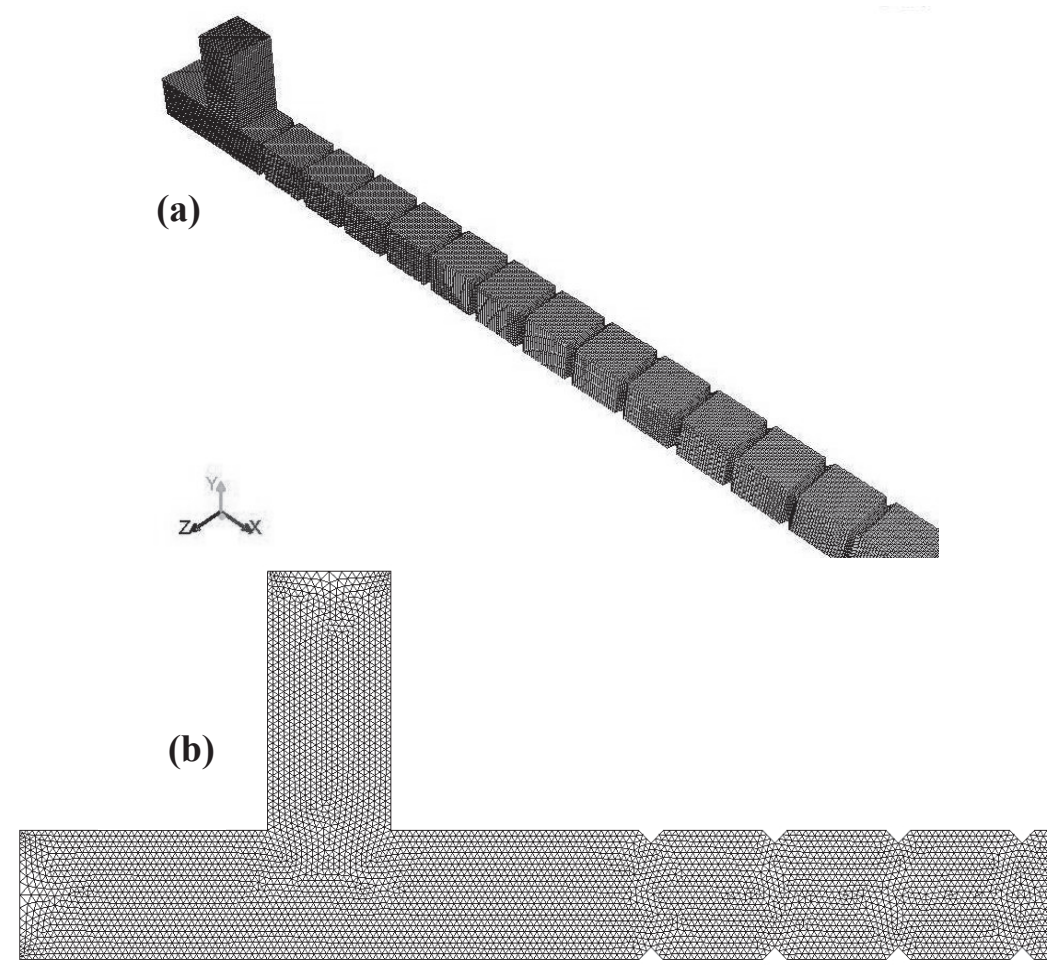

Fig. 2 - Mesh used for simulation in T-junction microchannel (a) isometric view (b) side view 


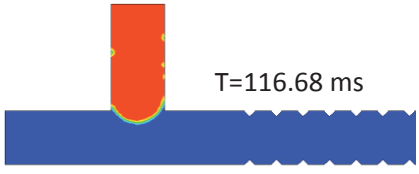

(a)

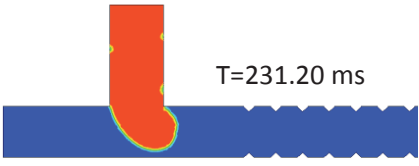

(c)

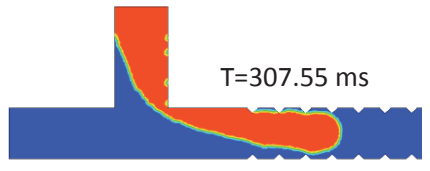

(e)

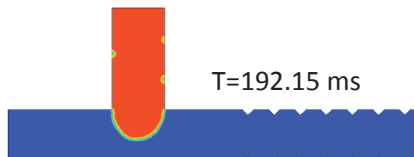

(b)

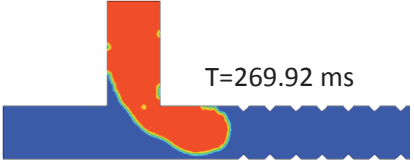

(d)

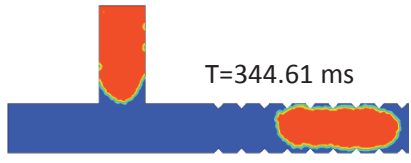

(f)

Fig. 3 - Six stages of slug formation $\left(u_{w}=0.05 \mathrm{~m} \mathrm{~s}^{-1}\right.$ and $\left.u_{c}=0.009 \mathrm{~m} \mathrm{~s}^{-1}, \mathrm{~s}=1 \mathrm{~mm}, \varepsilon=0.1 \mathrm{~mm}\right)$

$6.74 \times 10^{5}$, and $9.58 \times 10^{5}$ grids were tested for the grid independency and it was found that 674120 grids arrangement gives satisfactory results for velocity and pressure profile, displayed in Fig. 2(a) and (b). The boundary conditions velocity inlet was applied for the water and cyclohexane feed and specified as uniform entrance velocity, and pressure outlet condition was set at the outlet. The physical properties and simulation parameters used in the investigation are listed in Table 1.

The volume of fluid method (VOF) on Eulerian grids was employed to track the interface of the two immiscible fluids by solving an additional equation for the volume fraction. The Geometric Reconstruction technique was used for interface reconstruction and implicit body force treatment for the body force formulation. For unsteady state simulation, a segregated time-dependent solver was employed, and for discretization the PRESTO! (Pressure staggering option) scheme was used for pressure interpolation. The second-order upwind differencing scheme was used for solving transport equations, and PISO (pressure-implicit with splitting of operators) scheme was applied for pressure velocity coupling, which reduces the internal iteration per time step and allows larger under relaxation parameters. The absolute convergence criterion for the continuity equation and the velocity was 0.001 . The simulations were carried out on a desktop computer (Intel i7 CPU, $3.40 \mathrm{GHz}$ and $4 \mathrm{~GB}$ RAM). The typical simulation time for a mesh with 674000 cells was about 7-8 days. The maximum Courant number $\left(C_{r}\right)$ of 0.25 was used for the volume fraction calculation, which is defined as

$$
C_{r}=U \frac{\Delta t}{\Delta l}
$$

where, $U, \Delta t$ and $\Delta l$ are average flow velocity (total flow rate/cross-sectional area), maximum time steps size, and mesh size, respectively.

\section{Results and discussion}

\section{Slug generation mechanism}

The mechanism of dispersed-phase slug formation is illustrated in Fig. 3. The figure shows that the dispersed phase enters the main channel of continuous phase from the side entry, and forms a well-defined slug flow in the channel. Initially, the dispersed phase goes into the main stream and the drop begins to grow, Fig. 3( $a$ and $b$ ). The tip of the dispersed phase grows and blocks almost the entire cross-section of the main channel, and builds pressure in the upstream side. However, the dimensions of the channel limit the radius of the tip curvature, Fig. 3(c and d). The pressure builds up in the upstream side, forces the continuous phase fluid to flow in the gap between the wall and drop (dispersed phase) with higher flow velocity. Due to this shear exerted on the droplet, it elongates downstream and thus pressure decreases along the length of the channel. When the droplet enters the enhanced channel section, the droplet cross-section reduces and acquires the annulus region due to presence of obstructions. Whereas, continuous phase film thickness increases between the droplet and wall due to reduction in droplet cross-section. Further, the pressure downstream decreases and fluctuates around the obstruction element due to converging-diverging cross-section. On the other hand, in the mixing zone, the droplet interface moves toward the downstream, and the neck connected to the inlet of the dispersed phase becomes thinner, Fig. 3(e). Finally, the neck of the dispersed phase liquid is squeezed, and the slug separates and flows downstream, Fig. 3(f).

\section{Pressure variation}

Fig. 4( $a$ and $b$ ) shows the pressure variation in the continuous phase inlet and the dispersed phase 

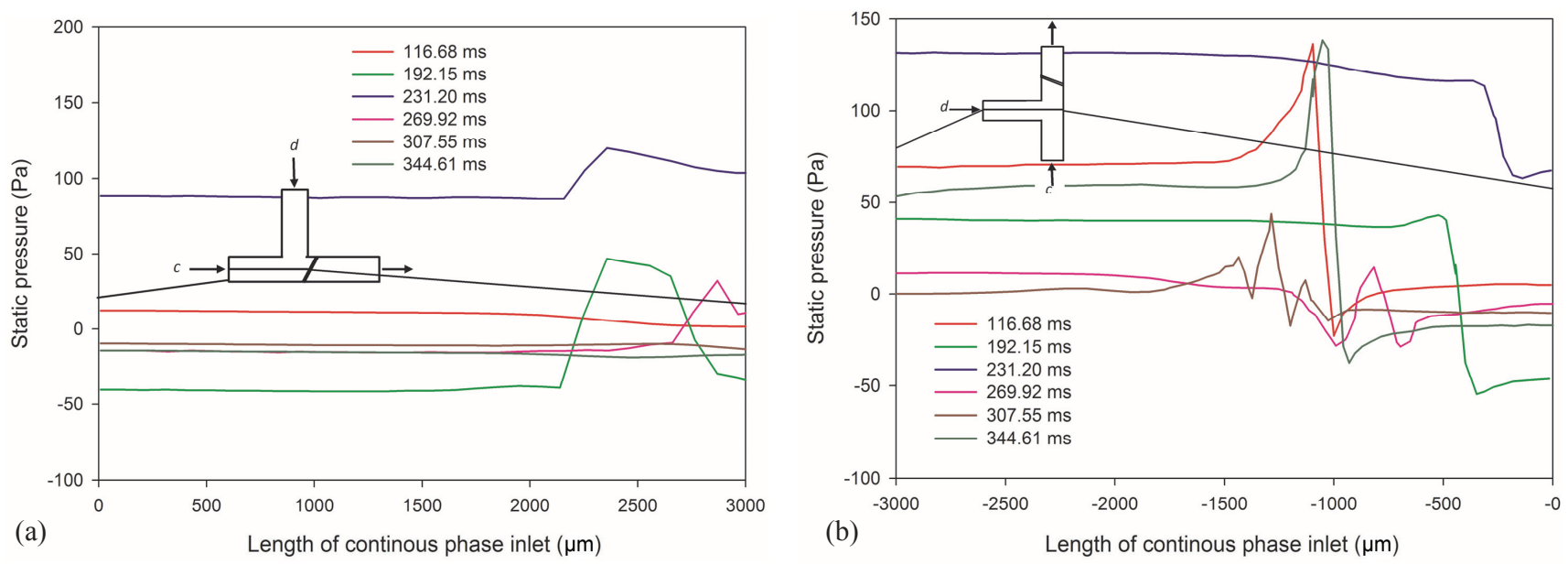

Fig. 4 - Static pressure behavior in the T-junction for six steps of slug formation (a) along the length of continuous-phase channel, and (b) along the length of dispersed-phase inlet $\left(u_{w}=0.05 \mathrm{~m} \mathrm{~s}^{-1}\right.$ and $\left.u_{c}=0.009 \mathrm{~m} \mathrm{~s}^{-1}, \mathrm{~s}=1 \mathrm{~mm}, \varepsilon=0.1 \mathrm{~mm}\right)$

inlet. In the early stage of slug formation, three forces are acting on the tip of the dispersed phase droplets, the force arising from increased resistance to flow of the dispersed phase fluid around the tip, the interfacial tension force, and the shear stress force of the continuous phase. These are elucidated as follows: In the first stage, dispersed-phase fluid goes into mainstream and the drop begins to grow, the pressure in the continuous phase inlet is lower in the upstream part, and increases in the vicinity of the T-junction due to the decrease in the flow cross-section. When the pressures in continuous phase and dispersed phase are compared near the T-junction, the former (Fig. 3a) is relatively lower compared to the latter (Fig. 3b). When the droplet occupies the entire cross-section of the channel, (Fig. 3c), high pressure is built up in the upstream region. Due to this, the droplet elongates and grows downstream, and the pressure decreases (Fig. 3d and e). These pressure fluctuations in the continuous phase and dispersed phase are due to cross-sectional area variation in downstream side, which periodically changes the pressure in the upstream part, and pressure fluctuation achieves an average value in the case of well-defined slug flow. The pressure in the dispersed-phase inlet fluctuates during all stages of the slug flow formation due to pressure fluctuation in continuous phase (Fig. 4b).

The Laplace pressure computed from the average pressure drop across the front interface and the back interface of droplet is plotted in Fig. 5. The points were randomly selected across the interface in the defined area and the average value was determined considering pressure drops at three positions: dispersed-phase inlet, across the entire emerging droplet, and across the separated slugs. The pressure drop at the dispersed-phase inlet clearly indicates the generation of slug flow. As the droplet penetrates the mainstream, the Laplace pressure de- creases since pressure in the continuous phase increases, which changes the curvature shape from concave to convex. In the third stage, where pressure in the continuous phase reaches maximum value, the Laplace pressure shows minimum value. As the neck of the dispersed phase breaks, the tip of the dispersed phase shrinks back to the inlet channel, and thus, the pressure in the continuous phase drops and Laplace pressure increases. In addition, pressure in the upstream side fluctuates and achieves an average value due to variation in channel cross-section downstream.

Fig. 5 depicts the variation of Laplace pressure with steps of slug flow generation. The figure clearly shows a subsequently decreasing behavior in pressure in step (b), (c), (d), and (e), when the Laplace pressure is computed across the emerging droplet. The reason is that, initially, the pressure at the tip of emerging droplet increases, and the drop-

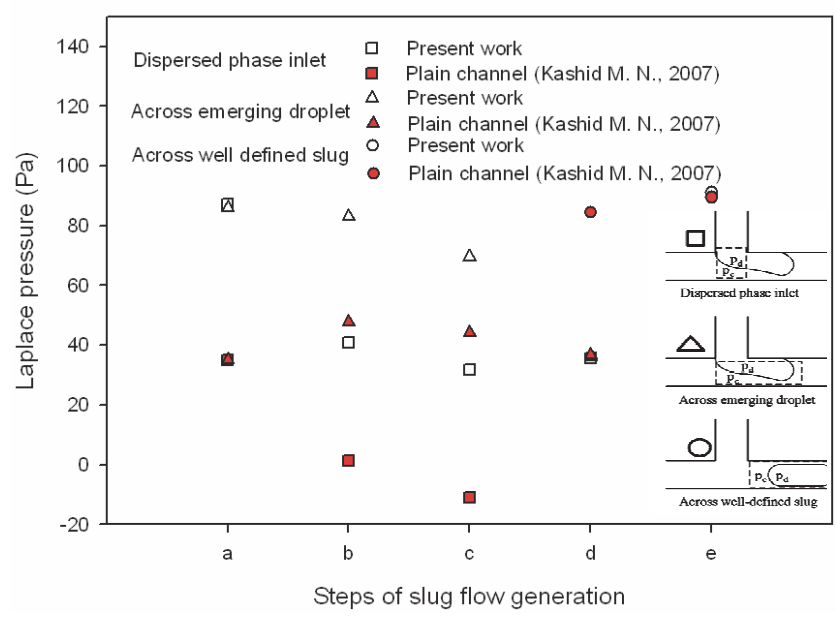

Fig. 5-Laplace pressure (difference between pressure in continuous ( $p$ ) and in dispersed ( $\left.p_{d}\right)$ phase) in different steps of slug flow formation $\left(u_{w}=0.05 \mathrm{~m} \mathrm{~s}^{-1}\right.$ and $u_{c}=0.009 \mathrm{~m} \mathrm{~s}^{-1}, \mathrm{~s}=$ $1 \mathrm{~mm}, \varepsilon=0.1 \mathrm{~mm}$ ). The Laplace pressure was calculated over the marked domain. 
let blocks almost the entire cross-sectional area of the main channel in the mixing zone, thus building up the pressure in the upstream side. When the droplet reaches the enhanced channel section, the droplet diameter reduces due to the presence of obstruction elements, and the fluid velocity at the tip of the emerging droplet increases while the pressure decreases. Therefore, Laplace pressure decreases in steps (b), (c), (d), and (e). The Laplace pressure for dispersed-phase inlet and across the emerging droplet is identical in steps (a) and (d), because in both cases, the droplet under investigation penetrates the continuous flow stream, i.e. the pressure points are similar. In the case of well-defined slug flow, the average pressure drop across the first droplet separated in step (e) is predicted. Each slug gives a pressure drop around $100 \mathrm{~Pa}$ in the well-defined slug flow. The predicted Laplace pressures for enhanced microchannel in this investigation were in good agreement with the experimentally obtained data of Kashid M. N. ${ }^{26}$ for plain microchannel, except for the slightly different behavior in the downstream, i.e. the enhanced section.

A clearer picture of the pressure behavior in a well-defined slug flow is given in Fig. 6. The pressure in the dispersed phase shows higher value compared to the continuous phase, and the difference between the values is the Laplace pressure. Consequently, the overall pressure in the liquid-liquid slug flow is due to three factors: pressure drop due to viscous faces, pressure drop due to obstruction element, and pressure drop due to interfacial tension (Laplace pressure). The predicted and experimental overall pressure variations were also in good agreement with the measurement of Kashid M. N. ${ }^{26}$ for plain microchannel. The figure also shows that predicted pressure in the enhanced microchannel is a function of obstruction spacing. The pressure drop decreases as the obstruction spacing increases. In fact, for smaller values of spacing, the viscous force is higher since a thick layer of cyclohexane is formed in between the obstruction elements compared to large spacing, while the flow of continuous phase is hindered by the obstruction elements. However, as obstruction spacing is increased, the flow resistance decreases.

The effect of obstruction spacing on the pressure drop gradient in the microchannel was investigated by performing a simulation for six different obstruction spacings, Table 1 , for $Q_{W} / Q_{C H}$ ratio of 0.18 . The predicted pressure drop gradient is shown in Fig. 7. As shown in Fig. 7, the pressure drop decreases with increased spacing until $s<1 \mathrm{~mm}$. In the range of $s$ from $1 \mathrm{~mm}$ to $1.25 \mathrm{~mm}$, the pressure drop increases and reaches maximum value at $s=$ $1.25 \mathrm{~mm}$. Thereafter, it again decreases to $s>1.25$ $\mathrm{mm}$. For smaller values of spacing, it was observed that the hydraulic diameter of the channel in downstream was smaller than the channel cross-section at the inlet. However, the hydraulic diameter (in downstream) increases with obstruction spacing. Therefore, the viscous force is higher in smaller obstruction spacing compared to larger spacing. In addition, the continuous-phase (cyclohexane) flow is also hindered by the obstruction elements. This is because the capillary force is slightly greater and overall pressure drop is quiet high for small spacing. The pressure drop decreases with increased obstruction spacing due to lower capillary force. Further, $s$ from $1 \mathrm{~mm}$, pressure drop increases and attains maximum value at $s=1.25 \mathrm{~mm}$. This is because a spherical cavity of hydraulic diameter $1 \mathrm{~mm}$ was observed between the adjacent obstruction elements; in this cavity, the slug expands and displaces the surrounding continuous-phase fluid occupying the maximum cross-section. Thus, capillary resistance increases because of a thin cyclohexane film present between the slug and channel wall. The

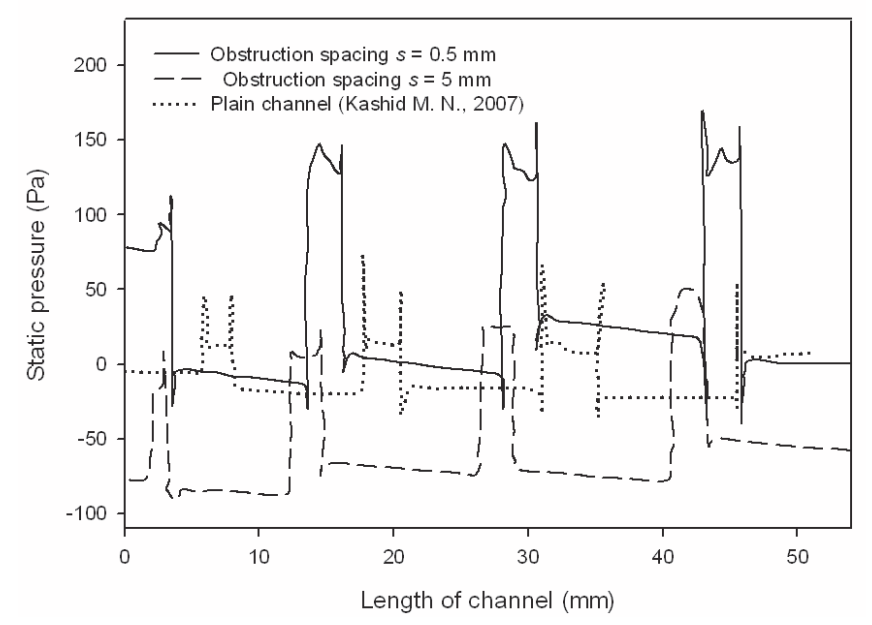

Fig. 6 - Static pressure behavior along the length of the channel in well-defined slug flow system $\left(u_{w}=0.05 \mathrm{~m} \mathrm{~s}^{-1}\right.$ and $u_{c}=0.009 \mathrm{~m} \mathrm{~s}^{-1}, s=1 \mathrm{~mm}, \varepsilon=0.1 \mathrm{~mm}$ )

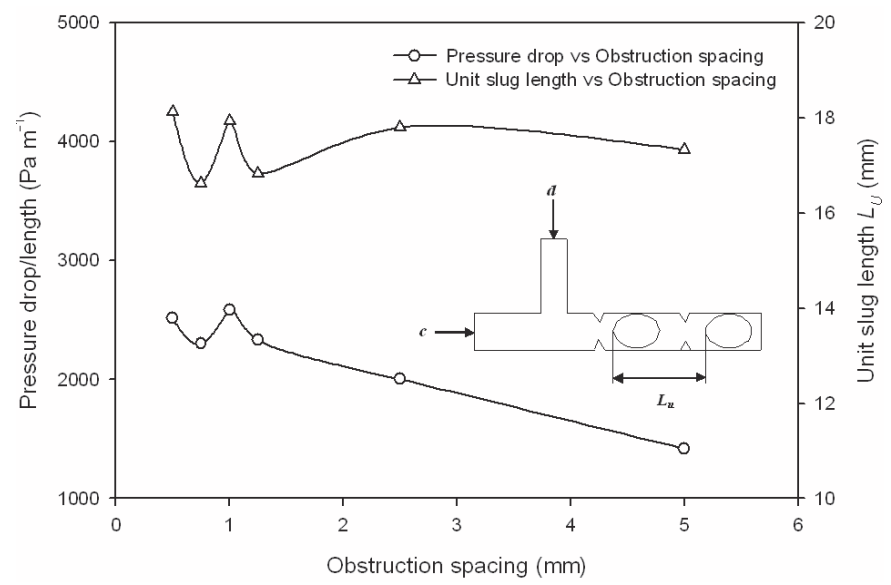

Fig. 7 - Two-phase pressure drop per unit length and unit slug length of water-cyclohexane slug flow versus spacing of obstruction elements (s) 
number of obstruction elements in a channel is larger for smaller values of spacing. Therefore, the pressure drop per unit length is larger for smaller values of spacing, and it decreases as the obstruction spacing increases. Fig. 7 also illustrates the effect of obstruction elements spacing on unit cell length. It was observed that the unit cell length varied with obstruction spacing in wavy form instead of smooth monotonic variation. It was found that unit cell length decreases-increases periodically, and is slightly larger for smaller spacing $(\sim 1 \mathrm{~mm})$ compared to larger spacing ( $\sim 5 \mathrm{~mm})$.

The effect of slug velocity on pressure drop was investigated by performing the simulation for T-junction enhanced microchannel $(s=5 \mathrm{~mm})$. The predicted pressure drop is shown in Fig. 8 for equal flow rates of both phases. A quantitative comparison between predicted pressure drops for enhanced channel with experimental/theoretical pressure drop for plain channel of Kashid M. N., is also shown in Fig. 8. The pressure drop for both cases was found to be dependent on slug velocity, and the predicted pressure drops were in a good agreement with his data with absolute deviations in the range of $10 \%$ to $20 \%$. The predicted pressure drop shows lower value compared to experimental/theoretical data due to the presence of a continuous-phase film, which provides a lubricating action on the embedded slug and yields annular flow behavior, exhibiting different pressure drop characteristics compared to the plain channel. Another possible explanation for this discrepancy is the internal circulation induced within each individual slug. When the slugs move through the channel, internal circulation arises within the slug, due to the shear between the channel wall and the axis of the slug, which eventually affects the capillary pressure. However, such circulation is advantageous in mass transfer as it enhances the diffusive penetration between the phases.

\section{Slug length variation}

Fig. 9 shows the dispersed-phase slug length as a function of obstruction elements spacing. As shown in Fig. 9, dispersed-phase slug length also exhibits wavy variation with obstruction spacing. In the enhanced microchannel, for smaller values of spacing, capillary resistance is higher and dispersed phase (water) experienced less shear force exerted by the continuous fluid due to thicker film of continuous phase. In addition, cross-sectional size of slug reduces due to small hydraulic diameter in the downstream (enhanced section), therefore fluid velocity at the droplet tip increases and the neck connecting it to the dispersed-phase inlet is squeezed early, and the slug separates and flows downstream. Whereas, for large obstruction spacing, a thin film of continuous phase is formed between the slug and wall, and the upstream fluid exerts higher shear force on the dispersed-phase slug. Therefore, the drop elongates and grows slowly in the downstream and a large slug is formed in the main channel.

In order to study the effect of scaling-up the slug size, simulations were carried out for different flow-rate ratios $\left(Q_{W} / Q_{C H}\right)$ ranging from 0.1 to 7 at low flow velocity of cyclohexane. The results of CFD simulation were again compared, and were found to be in good agreement with the experimental data, as shown in Fig. 10. It was observed that at $Q_{C H}=9 \mu \mathrm{L} \mathrm{s}^{-1}\left(\mathrm{Ca}=1.767 \cdot 10^{-4}\right)$, the viscous force is insignificant. In fact, the slug length is independent only on $Q_{W}$, and increases with increasing $Q_{W}$. A quantitative comparison of the measured and predicted slug lengths for $Q_{W} / Q_{C H}<2$ is also shown in Fig. 10. It can be seen that predicted slug length in enhanced microchannel $(s=1 \mathrm{~mm})$ is larger than that in plain channel. The reason being that the ob-

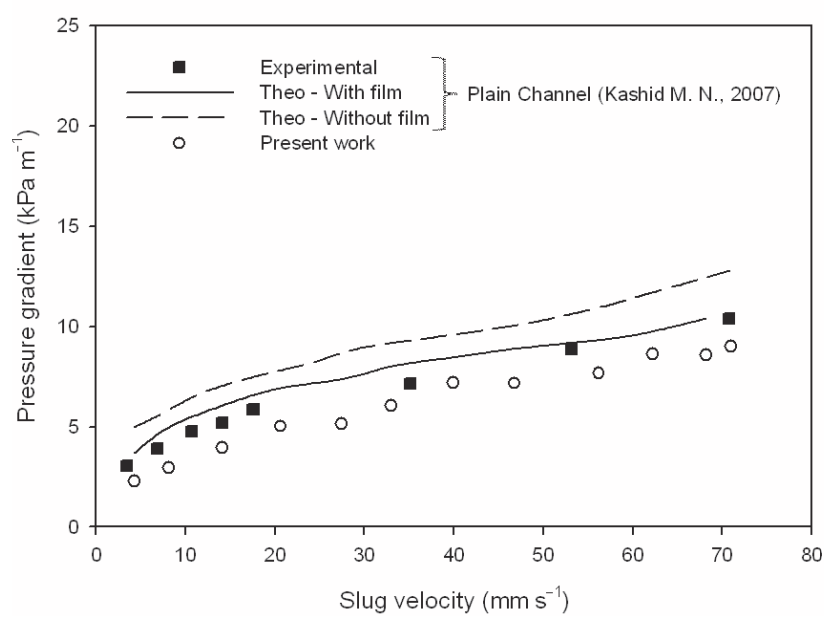

Fig. 8-Comparison of predicted and experimental pressure drop at equal volumetric flow rates of water and cyclohexane

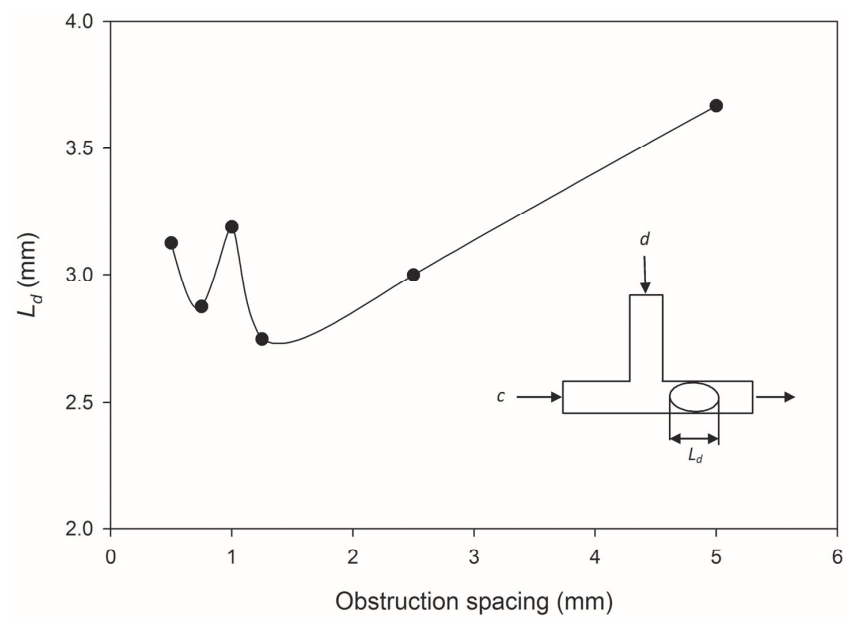

Fig. 9 - Length of dispersed-phase slug $\left(L_{d}\right)$ as a function of obstruction spacing (s) 


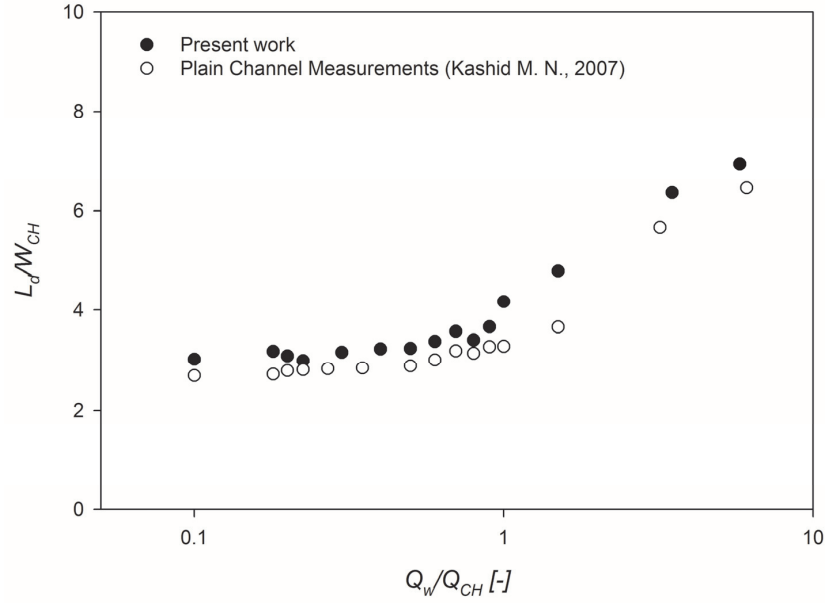

Fig. 10 - Effect of $Q_{W}$ on the slug length at a constant $Q_{C H}$ value of $9 \mu \mathrm{L} \mathrm{s}^{-1}$

struction element present in the enhanced microchannel significantly reduced the capillary number (Ca) of the continuous phase flow. Therefore, the role of viscous force becomes insignificant while the slug formation mechanism is governed by interfacial tension force, which causes formation of a long slug.

Fig. 11 shows the pressure gradient in the enhanced microchannel as a function of obstruction height. As illustrated in the figure, the pressure drop increases with the increase in obstruction height. The reason being that, for large obstruction height, the hydraulic diameter of downstream is less compared to the inlet for slug flow. Therefore, higher obstruction elements obstruct the continuous-phase

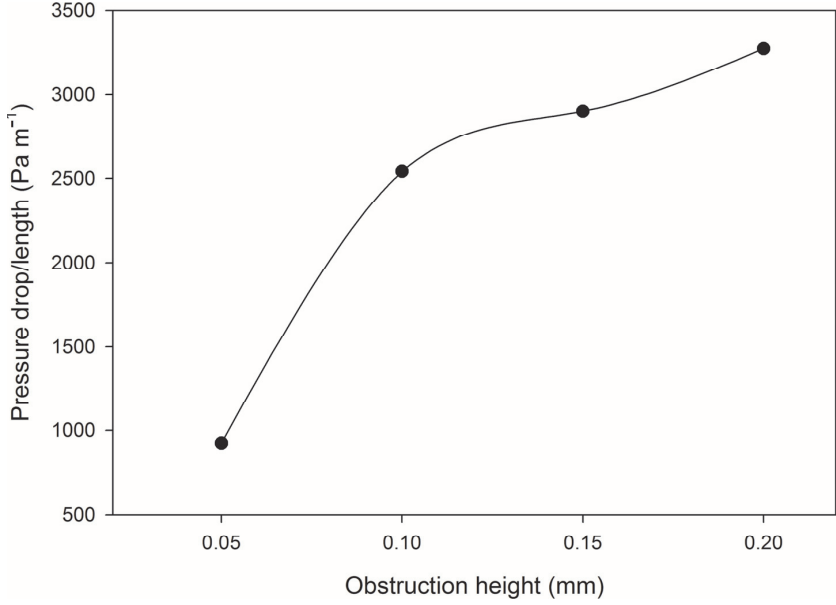

Fig. 11 - Two-phase pressure drop per unit length versus height of obstruction elements

(cyclohexane) flow, and form a thick liquid film in between the slug and channel wall, which diminishes capillary resistance more as compared to a thinner film. On the other hand, for small obstruction height, the hydraulic diameter of downstream is comparable to that of the inlet. In addition, viscous and capillary forces are much greater for higher obstruction elements compared to smaller height, and hence the net increase in pressure drop was observed with increasing obstruction height.

The velocity vectors and velocity contour for six different obstruction spacings in T-junction enhanced microchannel are shown in Fig. 12. It can be seen that the velocity of the backflow increases in the vicinity of the obstruction elements relative to
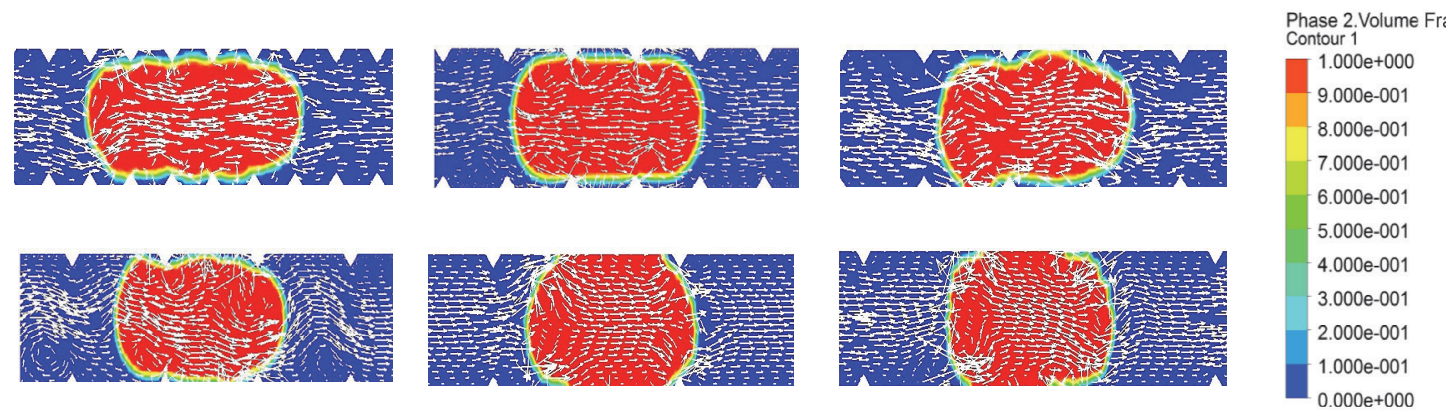

Velocity
Contour
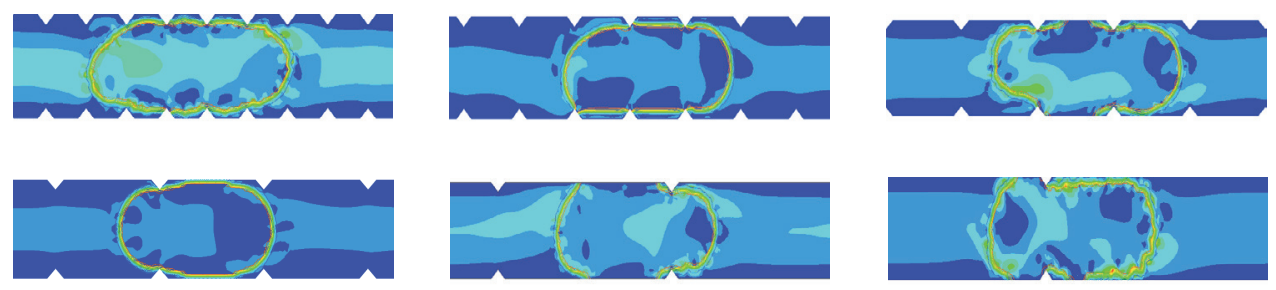

Fig. 12 - Velocity distribution within the slug. Internal circulation within the slug is represented by (a) velocity vector, (b) velocity contour $\left(u_{w}=0.05 \mathrm{~m} \mathrm{~s}^{-1}\right.$ and $\left.u_{c}=0.009 \mathrm{~m} \mathrm{~s}^{-1}\right)$.

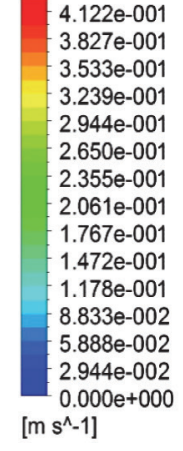


the flow rate close to the symmetry axis. Fluid particles near the obstruction elements flow in reverse direction and form eddies, as seen in Fig. 12(a) and 12(b). However, eddies formation was found to decrease with increased obstruction spacing. It was also observed that internal circulation at the front end and near the obstruction elements decreased with increased obstruction spacing. In addition, internal circulation at the front end and near the obstruction elements increased with obstruction height at the penalty of pressure drop. The velocity contour plots for slug flow in T-junction enhanced microchannel, Fig. 12(b), shows the circulation patterns and stagnant zones (zero vector magnitude) within the continuous and dispersed phase slugs. It is clear from the figure that the fluid mixing in the enhanced channel is greater compared to that in the smooth channel. Therefore this type of channel can improve the heat transfer from the channel walls and mass transfer between the fluids.

\section{Conclusion}

The present numerical study aimed to provide a volume of fluid (VOF)-based methodology to study the slug flow generation for liquid-liquid systems in a T-type enhanced microchannel, and predictions were validated using the measurement of Kashid M. $\mathrm{N} .{ }^{26}$ It was observed that the VOF method described the slug flow generation in enhanced channel quite accurately, along with the key characteristics, such as wall film and pressure drop of the channel. The pressure drop was found to be affected by the slug velocity, obstruction spacing and height in the enhanced channel. In addition, It was also observed that obstruction spacing in the enhanced channel affected the unit cell length and slug formation time. Further, for smaller obstruction spacing, flow resistance was slightly greater and decreased with increased obstruction spacing. Thus, fewer slugs had formed at smaller spacing. Furthermore, unit cell length and slug formation time were also slightly greater for smaller spacing and decreased with increased obstruction spacing. It was also found that water slug shape was affected by the obstruction spacing and height. The velocity vector and velocity contour plots indicated that the enhanced channel induced more internal mixing within a slug, rendering it ideal for enhancement of heat transfer and interface mass transfer.

\section{Nomenclature \\ $\mathrm{Ca}$ - capillary number \\ $L \quad$ - slug length, mm \\ $n$ - normal direction}

$\hat{n}-$ unit surface normal vector

$p$ - pressure, $\mathrm{Pa}$

$Q \quad$ - volumetric flow rate, $\mu \mathrm{L} \mathrm{s}^{-1}$

$s \quad-$ obstruction spacing, $\mathrm{mm}$

$s_{0} \quad-$ width of obstruction element, $\mathrm{mm}$

$t \quad-$ time, $\mathrm{s}$

$u \quad$ - velocity, $\mathrm{m} \mathrm{s}^{-1}$

$W \quad$ - width of the inlet, $\mathrm{mm}$

$$
\begin{array}{ll}
\text { Greek letters } \\
\begin{array}{ll}
\alpha & - \text { marker function } \\
\mu & - \text { viscosity } \\
\kappa_{n} & - \text { divergence of unit normal } \\
\sigma & - \text { surface tension } \\
\rho & - \text { density } \\
\phi & - \text { volume fraction } \\
\varepsilon & - \text { height of obstruction element }
\end{array}
\end{array}
$$

\section{References}

1. Stankiewicz, A. I., Moulijn, J. A., Process intensification: Transforming chemical engineering, Chem. Eng. Prog. 96 (2000) 22

2. Ehrfeld, W., Hessel, V., Löwe, H., State of the art of microreaction technology. Microreactors: New Technology for Modern Chemistry, Wiley-VCH, Weinheim, 2000, pp 1-14.

3. Jähnisch, K., Hessel, V., Löwe, H., Baerns, M., Chemistry in microstructured reactors, Angew. Chem. Int. Ed. 43 (2004) 406.

doi: http://dx.doi.org/10.1002/anie.200300577

4. Talimi, V., Muzychka, Y. S., Kocabiyik, S., A review on numerical studies of slug flow hydrodynamics and heat transfer in microtubes and microchannels. Int. J. Multiphase Flow 39 (2012) 88

doi: http://dx.doi.org/10.1016/j.ijmultiphaseflow.2011.10.005

5. Dessimoza, A.-L., Cavinb, L., Renkena, A., Kiwi-Minskera, $L$., Liquid-liquid two-phase flow patterns and mass transfer characteristics in rectangular glass microreactors, Chem. Eng. Sci. 63 (2008) 4035. doi: http://dx.doi.org/10.1016/j.ces.2008.05.005

6. Triplett, K. A., Ghiaasiaan, S. M., Abdel-Khalik, S. I., Sadowski, D. L., Gas-liquid two-phase flow in microchannels, Part I: two-phase flow pattern, Int. J. Multiphase Flow 25 (1999) 377. doi: http://dx.doi.org/10.1016/S0301-9322(98)00054-8

7. Xu, J. L., Cheng, P., Zhao, T. S., Gas-liquid two-phase flow regimes in rectangular channels with mini/microgaps, Int. J. Multiphase Flow 25 (1999) 411.

doi: http://dx.doi.org/10.1016/S0301-9322(98)00057-3 
8. Yu, Z., Hemminger, O., Fan, L. S., Experiment and lattice Boltzmann simulation of two-phase gas-liquid flows in microchannels, Chem. Eng. Sci. 62 (2007) 7172. doi: http://dx.doi.org/10.1016/j.ces.2007.08.075

9. Takamasa, T., Hazuku, T., Hibiki, T., Experimental study of gas-liquid two-phase flow affected by wall surface wettability, Int. J. Heat Fluid Flow 29 (2008) 1593. doi: http://dx.doi.org/10.1016/j.ijheatfluidflow.2008.09.001

10. Donata, M. F., Franz, T., Philipp, R. V. R., Segmented gas-liquid flow characterization in rectangular microchannels, Int. J. Multiphase Flow 34 (2008) 1108. doi: http://dx.doi.org/10.1016/j.ijmultiphaseflow.2008.07.002

11. Wang, K., Lu, Y. C., Xu, J. H., Luo, G. S.; Determination of dynamic interfacial tension and its effect on droplet formation in the T-shaped microdispersion process, Langmuir $\mathbf{2 5}$ (2009) 2153. doi: http://dx.doi.org/10.1021/la803049s

12. Kashid, M. N., Agar, D. W., Turek, S., CFD modelling of mass transfer with and without chemical reaction in the liquid-liquid slug flow microreactor, Chem. Eng. Sci. 62 (2007) 5102.

doi: http://dx.doi.org/10.1016/j.ces.2007.01.068

13. Xu, J. H., Luo, G. S., Li, S. W., Chen, G. G., Shear force induced monodisperse droplet formation in a microfluidic device by controlling wetting properties, Lab Chip 6 (2006) 131.

doi: http://dx.doi.org/10.1039/B509939K

14. Zhao, Y. C., Ying, Y., Chen, G. W., Yuan, Q., Characterization of micromixing in T-shaped micromixer (in Chinese), J. Chem. Ind. Eng. 57 (2006) 1184.

15. Zhao, Y. C., Chen, G. W., Yuan, Q., Liquid-liquid two-phase flow patterns in a rectangular microchannel, AIChE J. 52 (2006) 4052. doi: http://dx.doi.org/10.1002/aic.11029

16. Zhao, Y. C., Chen, G. W., Yuan, Q., Liquid-liquid two-phase mass transfer in the T-junction microchannels, AIChE J. 53 (2007) 3042. doi: http://dx.doi.org/10.1002/aic.11333

17. Kobayashi, I., Mukataka, S., Nakajima, M., Production of monodisperse oil-in-water emulsions using a large silicon straight through microchannel plate, Ind. Eng. Chem. Res. 44 (2005) 5852.

doi: http://dx.doi.org/10.1021/ie050013r

18. Kashid, M. N., Platte, F., Agar, D. W., Turek, S., Computational modelling of slug flow in a capillary microreactor, J. Comput. Appl. Math. 203 (2007a) 487. doi: http://dx.doi.org/10.1016/j.cam.2006.04.010

19. Nisisako, T., Torii, T., Higuchi, T., Droplet formation in a microchannel network, Lab Chip 2 (2002) 24. doi: http://dx.doi.org/10.1039/B108740C

20. Thorsen, T., Roberts, R. W., Arnold, F. H., Quake, S. R., Dynamic pattern formation in a vesicle-generating microfluidic device, Phys. Rev. Lett. 86 (2001) 4163. doi: http://dx.doi.org/10.1103/PhysRevLett.86.4163

21. Garstecki, P., Feuerstman, M., Stone, H. A., Whitesides, G. $M$., Formation of droplets and bubbles in a microfluidic T-junction: scaling and mechanism of breakup, Lab Chip 6 (2006) 437. doi: http://dx.doi.org/10.1039/b510841a

22. Tice, J. D., Lyon, A. D., Ismagilov, R. F., Effects of viscosity on droplet formation and mixing in microfluidic channels, Anal. Chim. Acta. 507 (2004) 73. doi: http://dx.doi.org/10.1016/j.aca.2003.11.024

23. Kashid, M. N., Agar, D. W., Hydrodynamics of liquid-liquid slug flow capillary micro-reactor: flow regimes, slug size and pressure drop, Chem. Eng. J. 131 (2007) 1. doi: http://dx.doi.org/10.1016/j.cej.2006.11.020

24. Kashid, M. N., Renken, A., Kiwi-Minsker, L., CFD modelling of liquid-liquid multiphase microstructured reactor: Slug flow generation, Chem. Eng. Res. Des. 88 (2010) 362 doi: http://dx.doi.org/10.1016/j.cherd.2009.11.017

25. Yong, Y., Yang, C., Jiang, Y., Joshi, A., Shi, Y., Yin, X., Numerical simulation of immiscible liquid-liquid flowin microchannels using lattice Boltzmann method, Sci. China Chem. 54 (2011) 244 doi: http://dx.doi.org/10.1007/s11426-010-4164-z

26. Kashid, M. N., Experimental and modelling studies on liquid-liquid slug flow capillary microreactors, Biochemical and Chemical Engineering, Dortmund, University of Dortmund, Germany, 2007, pp 1-213. 